\title{
Erratum to: Variation of structural, optical, dielectric and magnetic properties of $\mathrm{SnO}_{2}$ nanoparticles
}

\author{
Zulfiqar $^{1,2} \cdot$ Rajwali Khan $^{3} \cdot$ Yuliang Yuan $^{1} \cdot$ Zainab Iqbal $^{4} \cdot$ Jie Yang $^{1} \cdot$ \\ Weicheng Wang ${ }^{1} \cdot$ Zhizhen $\mathrm{Ye}^{1} \cdot$ Jianguo $\mathrm{Lu}^{1}$
}

Published online: 27 July 2017

(C) Springer Science+Business Media, LLC 2017

\section{Erratum to: J Mater Sci: Mater Electron (2017) 28:4625-4636 DOI 10.1007/s10854-016-6101-1}

The original version of the article unfortunately contained errors in the equations, miscalculation and changes in figure. The detailed description of the error and corrections are given below.

The optical band-gap energy of a semiconductor can be derived from the reflectance spectrum. The reflectance $(R)$ is converted to absorbance $(F(R))$ using the Kubelka-Munk function,

$F(R)=\frac{(1-R)^{2}}{2 R}$

The band gap is determined by the $(F(R) \times E)^{2} \sim E$ curve with the extrapolated line at $(F(R) \times E)^{2}=0$, where $E$ is the incident photon energy $(h \nu)$.

To plot the curve, the data were processed in the origin software, in which Eq. (1) might be displayed as,

The online version of the original article can be found under doi:10.1007/s10854-016-6101-1.

Jianguo Lu

lujianguo@zju.edu.cn

1 State Key Laboratory of Silicon Materials, School of Materials Science and Engineering, Zhejiang University, Hangzhou 310027, China

2 Department of Physics, Abdul Wali Khan University, Mardan 23200, Khyber Pukhtunkhwa, Pakistan

3 Department Physics, Zhejiang University, Hangzhou 310027, China

4 Institute of Chemical Sciences, University of Peshawar, Peshawar, Khyber Pukhtunkhwa, Pakistan
$\operatorname{col}(E)=(1-\operatorname{col}(D))^{2} /(2 * \operatorname{col}(D))$

where $\operatorname{col}(\mathrm{E})$ and $\operatorname{col}(\mathrm{D})$ are $F(R)$ and $R$, respectively. However, due to Zulfiqar's carelessness, Eq. (2) was miswritten as,

$\operatorname{col}(E)=\left((1-\operatorname{col}(D))^{2} / 2 * \operatorname{col}(D)\right)$

Because of this mistake, the obtain $F(R) \sim \lambda$ and $(F(R) \times E)^{2} \sim E$ curves are wrong and the thus determined optical band-gap energies are incorrect.

Figure 8 appeared incorrectly in the published version of this article. The corrected figure is shown below. Based on the corrected figure, the optical band-gap energies are found to be 3.93, 3.98, 4.00, 4.01, and $4.04 \mathrm{eV}$ for $\mathrm{SnO}_{2}$ nanoparticles calcined at 400, 500, 600, 700, and $800^{\circ} \mathrm{C}$, respectively.

The analyses and conclusion will have to be corrected, accordingly. The details of changes are illustrated in this Erratum, with corrected texts listed as followings.

\begin{abstract}
The oxygen content increases steadily with increasing temperature, with the $\mathrm{Sn}: \mathrm{O}$ atomic ratio very near to the stoichiometric value of $1: 2$ at high temperatures suggesting the low density of defects. The optical band gap energies of $\mathrm{SnO}_{2}$ nanoparticles decrease from 4.04 to $3.93 \mathrm{eV}$ as the temperatures decrease, while room temperature ferromagnetism and electrical conductivity are enhanced with reduced temperatures. The presence of oxygen vacancies is confirmed by EDX, Raman, PL, XPS, and UV-Vis spectra. The band gap narrowing due to defects such as oxygen vacancies has been confirmed in $\mathrm{SnO}_{2}$ nanoparticles at low calcination temperatures. This visible light emission,
\end{abstract}


enhanced conductivity and room temperature ferromagnetism demonstrate that $\mathrm{SnO}_{2}$ nanoparticles may be promising in the visible light photo catalysis and optoelectronic devices.

\section{Introduction}

The 4th paragraph in the Introduction section:

Due to reduced defect density band gap increases along with increase in electrical conductivity and coercivity, while saturation magnetization reduces. The band gap energies of $\mathrm{SnO}_{2}$ nanoparticles are larger than bulk $\mathrm{SnO}_{2}$, but the band gap narrowing can be confirmed due to increase of defects at low temperatures. The visible light emission, enhancement in electrical conductivity and maximum saturation magnetization are very inspiring results. Oxygen vacancies can lead to the band gap narrowing and enhanced room temperature ferromagnetism. These $\mathrm{SnO}_{2}$ nanoparticles have potential applications in the fields of optoelectronic devices in the visible light range.

\section{Results and discussions}

The 8th paragraph in the "Results and discussions" Section:

The optical band gap energy $\left(E_{\mathrm{g}}\right)$ is directly calculated from Kubelka-Munk equation, where $(F(R) \times E)^{2}$ is plotted against y-axis and the incident photon energy $E=h \nu$ on x-axis. $E_{\mathrm{g}}$ can be evaluated by extrapolation of the linear portion at $(F(R) \times E)^{2}=0$, as shown in the inset of Fig. 8b. Thus the determined $E_{\mathrm{g}}$ values are 3.93, 3.98, 4.00, 4.01, and $4.04 \mathrm{eV}$ for $\mathrm{SnO}_{2}$ nanoparticles calcined at 400, 500, 600,700 , and $800^{\circ} \mathrm{C}$, respectively. The values are larger than the bulk $\mathrm{SnO}_{2}(3.6 \mathrm{eV})$. Clearly, a slight red shift of the band gap is noticed with decreasing calcination temperature [32, 33], as shown in Fig. 8b. This means that the band gap becomes narrow as decreasing temperatures. The band-gap narrowing is mainly due to the large number of defects (e.g., oxygen vacancies and tin interstitials) in $\mathrm{SnO}_{2}$ nanoparticles calcined at low temperatures. The colour of samples calcined at higher temperatures becomes more and more white, which suggests the concentration of oxygen vacancies decreases with increasing calcination temperature.

\section{Conclusion}

With increasing calcination temperature $\mathrm{Sn}^{2+}$ oxidized to $\mathrm{Sn}^{4+}$ and as a result the concentration of oxygen vacancies

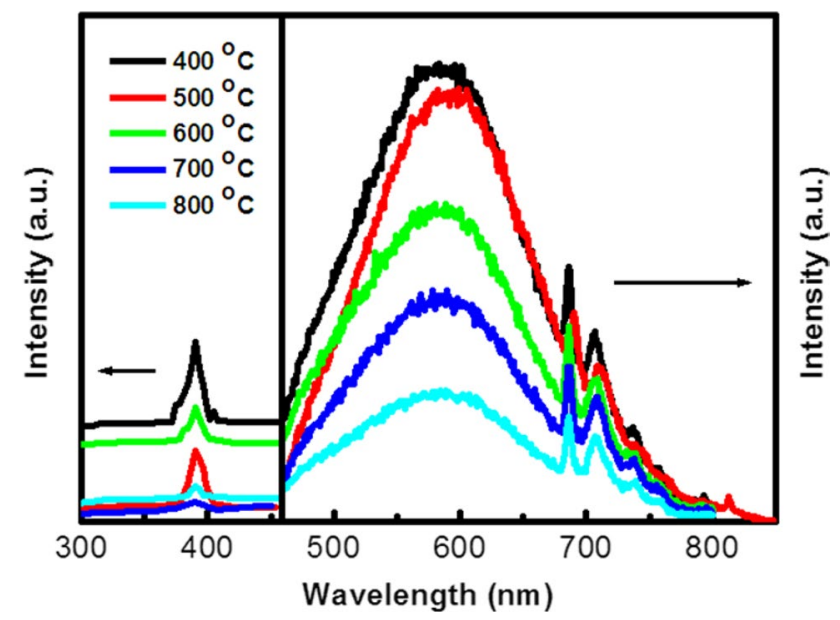

Fig. 7 PL spectra of $\mathrm{SnO}_{2}$ nanoparticles at various calcinations temperatures

decreases as confirmed by EDX, Raman, UV-Vis and XPS. The band gap values of $\mathrm{SnO}_{2}$ nanoparticles are larger than the bulk $\mathrm{SnO}_{2}$. The narrowed band gap, increased emission intensities, enhanced conductivity and room temperature ferromagnetism are obtained at low temperature, which is induced by the presence of large number of crystal defects like oxygen vacancies in nanocrystals. At high temperature, the crystallanity is enhanced with grain growth and low density of defects. The band gap is enlarged to be up to $4.04 \mathrm{eV}$ and the conductivity is increased due to enhancement in the volume of conductive grains, while room temperature ferromagnetism with reduced Ms and enhanced $\mathrm{Hc}$ are due to reduction in concentration of oxygen vacancies at higher calcination temperatures. The enhanced visible light emission, enhanced conductivity and room temperature ferromagnetism of $\mathrm{SnO}_{2}$ nanoparticles will open the door of applications in the fields of catalysis, sensors, and various optoelectronic devices. Also, this method can be extended to other wide band gap metal oxides to enhance their visible light emission for effectively making use of visible light.

In addition, the PL spectra were measured at different time in the wavelength ranges of $300-460$ and $460-800 \mathrm{~nm}$, respectively. Figure 7 appeared inappropriately in the published version of this article. The corrected figure is shown above. The change of the PL spectra has no influence on the text in the original article. 

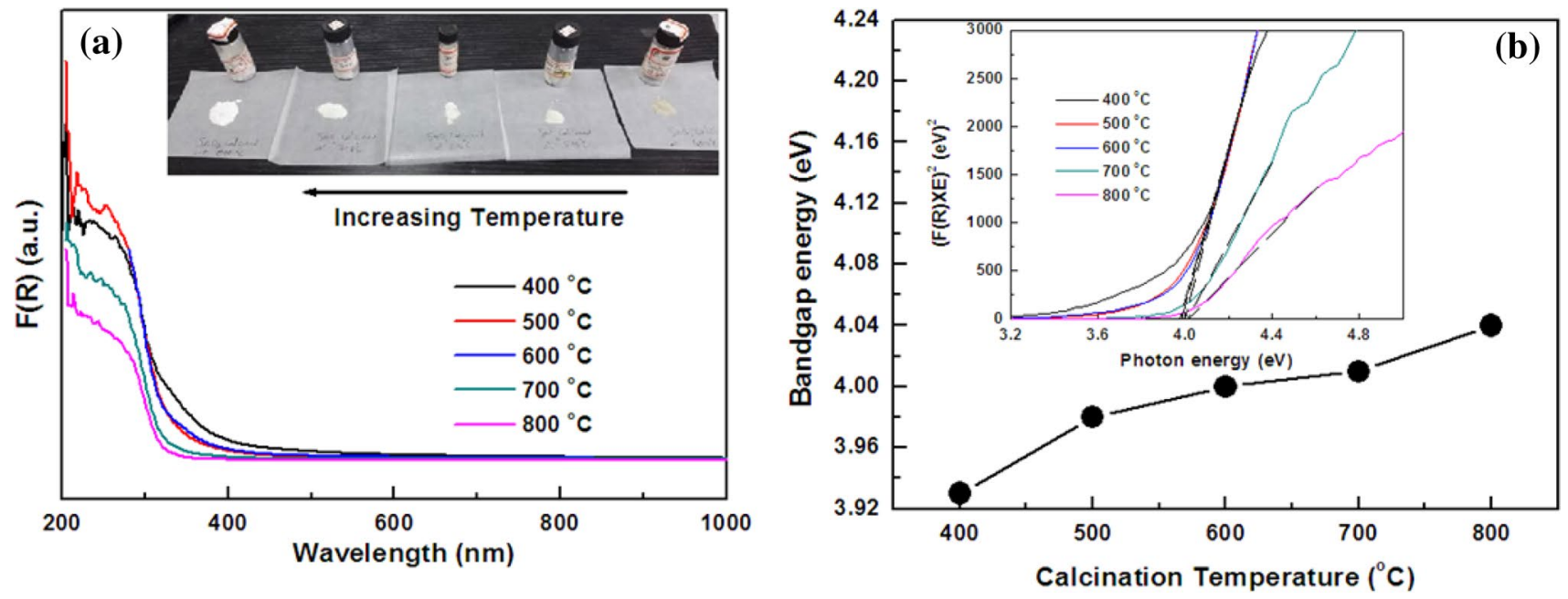

Fig. 8 a Absorbance spectra of $\mathrm{SnO}_{2}$ nanoparticles at various calcination temperatures. b Optical bandgap energies of $\mathrm{SnO}_{2}$ nanoparticles as a function of calcination temperature, with the inset showing the plots of $(F(R) \times E)^{2}$ versus photon energy $(E)$ 